Maria Walsh

\title{
Übergangsmanagement bei Haftentlassung aus dem bayerischen Jugendstrafvollzug
}

\author{
Zur Umsetzung der gesetzlichen Vorgaben in der Praxis
}

\begin{abstract}
Das Thema „Übergangsmanagement“ erfreut sich großer Beliebtheit, da zunehmend Mechanismen in die Haftentlassung eingebaut werden sollen, um dem so genannten „Entlassungsloch “1 entgegenzuwirken. Was sich in der Theorie vielversprechend anhört, könnte jedoch in der Praxis an organisatorischen, personellen oder Schnittstellenproblemen scheitern. Der folgende Beitrag behandelt die praktische Umsetzung der gesetzlichen Vorgaben zur Haftentlassung in Bayern am Beispiel der Entlassung aus dem Jugendstrafvollzug. Hierbei wird zunächst ein Überblick über die gesetzlichen Vorschriften gegeben, bevor näher auf den Prozess Übergangsmanagement und den Forschungsstand zur Haftentlassung eingegangen wird. Im Anschluss werden die Untersuchungen und deren Ergebnisse beschrieben, um schließlich die gesetzlichen Vorgaben in Anbetracht der Untersuchungsergebnisse zu diskutieren.

Schlïsselwörter: Übergangsmanagement, Entlassungsvorbereitung, Haftentlassung, Jugendstrafvollzug
\end{abstract}

Reentry Management and Release in Bavarian Juvenile Penal System - On the Application of Legal Requirements in Practice

Reentry management is a popular criminal policy issue, as evidenced by the growing desire to initiate mechanisms to belp prisoners reenter society. While the goal sounds promising, such attempts could fail due to organizational, personnel or cooperational issues. This article deals with a study on the practical application of legal requirements for reentry management and release of detainees from Bavarian juvenile penal system in practice. First, the legal requirements are outlined. Second, reentry management and the state of research on prison release are discussed. Third, the study and its findings are described. Lastly, the legal requirements and these findings are compared.

Keywords: Reentry management, release preparations, prison release, juvenile justice system, post-release experience

1 Matt NK 22 (2010), 34, 34.

NK 26. Jg. 3/2014 


\section{A. Einleitung}

Die Haftentlassung stellt für viele Inhaftierte eine Herausforderung dar, die mit zahlreichen Ängsten, Unsicherheiten und Problemen verbunden ist. ${ }^{2}$ Der Übergang zwischen dem unselbstständigen und strukturierten Haftalltag in ein selbst zu organisierendes Leben in Freiheit gestaltet sich für viele-insbesondere junge-Menschen schwierig. ${ }^{3}$ Zudem haben die Haftentlassenen mit Herausforderungen in verschiedenen Lebensbereichen zu kämpfen, die als kriminogene Faktoren ${ }^{4}$ wirken können. ${ }^{5}$ Oftmals wird auch die hohe Rückfallgefahr innerhalb der ersten sechs Monate nach Haftentlassung mit diesen Anpassungsschwierigkeiten in Verbindung gebracht. ${ }^{6}$

Ein erfolgreicher Übergang von der Inhaftierung in die Freiheit ist wichtiger Bestandteil des Strafvollzugs, da hierdurch die Vollzugsziele „Resozialisierung “ und „Sicherheit der Gesellschaft" verfolgt werden. Dem so genannten „Übergangsmanagement“" bei Haftentlassung kommt dabei eine besondere Bedeutung zu, auf das einige Bundesländer mit spezifischen Normen innerhalb ihrer Vollzugsgesetze reagiert haben. ${ }^{8}$

Der vorliegende Beitrag beschäftigt sich mit der praktischen Umsetzung der gesetzlichen Vorschriften zur Haftentlassung in Bayern am Beispiel der Entlassung aus dem Jugendstrafvollzug. Hierbei liegt der Fokus auf der Kooperation zwischen Justizvollzugsanstalten und Bewährungshilfe sowie der Beurteilung der Haftentlassungsvorbereitung aus Sicht einzelner Haftentlassener.

\section{B. Rechtliche Vorgaben zur Haftentlassung in Bayern}

Die Entlassungsvorbereitung für den Jugendstrafvollzug ist in Art. 136 BayStVollzG geregelt. Gemäß Art. 136 Abs. 1 Satz 1 BayStVollzG arbeiten die Jugendstrafvollzugsanstalten rechtzeitig vor dem voraussichtlichen Entlassungstermin mit vertrauenswürdigen Dritten und Institutionen außerhalb des Vollzugs zusammen, um zu erreichen, dass die jungen Gefangenen bei der Entlassung über eine geeignete Unterbringung und eine Arbeits- oder Ausbildungsstelle verfügen. Im Gegensatz zu den Vorschriften zur Haftentlassung bei erwachsenen Gefangen handelt es sich hierbei um eine Muss-Vorschrift. Soweit angeordnet, wird zudem die Bewährungshilfe unterrichtet (Art.138 Abs. 1 Satz 3 BayStVollzG). Weiterhin können den jungen Gefangenen im Rahmen der Vorbereitung der Entlassung verschiedene Vollzugslockerungen, wie Sonderurlaub oder Verlegung in den offenen Vollzug, gewährt werden (Art. 136 Abs. 3-5 BayStVollzG).

2 Hosser u.a. 2007, 405.

3 Bereswill 2007, 300.

4 Mangelnde Einbindung in den Leistungsbereich, Armut, Verschuldung, Substanzmittelmissbrauch, fehlendes soziales Netzwerk und defizitäre Sozialkompetenzen.

5 Schwedler 2013, 194.

6 Matt ZJJ 22 (2011), 422, 423; Löprick 2007, 436.

7 Übergangsmanagement umfasst die durchgeführten Maßnahmen zur Erleichterung des Wiedereintritts in die Gesellschaft (Arloth 2010, 346).

8 Arloth 2008, $3 \mathrm{f}$. 
Gemäß Art. 79 BayStVollzG soll den Gefangenen vor der Entlassung bei der Ordnung ihrer persönlichen, wirtschaftlichen und sozialen Angelegenheiten geholfen werden, wobei ein besonderes Augenmerk auf die Unterstützung bei der Suche nach Arbeit, Unterkunft und persönlichem Beistand gelegt werden soll.

Die Bekanntmachung des Bayerischen Staatsministeriums der Justiz für Bewährungshilfe, Führungsaufsicht und Gerichtshilfe (BewHBek) sieht vor, die Umstände, unter denen der Verurteilte künftig leben muss (Familienverhältnisse, Unterkunft, Arbeitsplatz u.a.), soweit möglich, vor Strafrestaussetzung zu klären (Nr. 7.1.1.1 Satz $1 \mathrm{BewH}$ Bek). Weiterhin kann gemäß Nr. 7.1.1.2 Satz 1 BewHBek im Fall einer zu erwartenden Aussetzung des Strafrestes und einer Unterstellung unter die Aufsicht eines Bewährungshelfers der für den künftigen Wohn- oder Aufenthaltsort zuständige Bewährungshelfer bei der Vorbereitung der Rückkehr des Verurteilten in die Freiheit eingeschaltet werden. Daher empfiehlt es sich, dass sich die Gerichte (Vollstreckungsleiter) in solchen Fällen bereits einige Zeit vor der Entscheidung über die Aussetzung mit dem zuständigen Bewährungshelfer in Verbindung setzen (Nr.7.1.1.2 Satz 2 BewHBek). Gemäß Nr. 7.1.1.2 Satz 4 BewHBek kann es angezeigt sein, dass der Bewährungshelfer zur Vorbereitung der Entlassung eines Verurteilten auch mit der Vollzugsanstalt oder über diese mit dem Verurteilten Kontakt aufnimmt. In welchen Fällen eine solche Kontaktaufnahme angezeigt ist, wird nicht definiert.

Bei der Kooperation zwischen Justizvollzugsanstalten und Bewährungshilfe vor der Haftentlassung handelt es sich durchweg um fakultative Regelungen mit einem Ermessensspielraum.

\section{C. Übergangsmanagement}

Das Übergangsmanagement soll es den Inhaftierten ermöglichen, die Anforderungen der schwierigen Haftentlassungssituation zu bewältigen. ${ }^{9}$ Dazu sollten sie sich im Rahmen des Übergangsmanagements mit verschiedenen Lebensbereichen sowie ihren kriminogenen und stabilisierenden Faktoren auseinandersetzen. ${ }^{10}$ Der Begriff Übergangsmanagement umfasst drei verschiedene Ansätze: (1) Strategien zur Verbesserung der Integration nach Haftentlassung bereits im Vorfeld, (2) Strukturierung des Haftübergangs und (3) Nachsorge. ${ }^{11}$

Die Deutsche Vereinigung für Jugendgerichte und Jugendgerichtshilfen fordert in ihren Mindeststandards für den Jugendstrafvollzug die Umsetzung eines solchen Übergangsmanagements. Unter anderem soll der Vollzug von Beginn der Haftstrafe an auf die Entlassung und Wiedereingliederung der Inhaftierten in die Gesellschaft ausgerichtet

9 Arloth 2010, $346 \mathrm{f}$.

10 Matt ZJJ 22 (2011), 422, 425.

11 Ders. 2011, 423.

NK 26. Jg. 3/2014 
sein. Spätestens sechs Monate vor der geplanten Entlassung sollte eine Entlassungsvorbereitung in Zusammenarbeit mit den Schnittstellen erfolgen. ${ }^{12}$

Im Falle einer Strafrestaussetzung sollte bereits während der Strafvollstreckung eine Kooperation zwischen der jeweiligen JVA und dem zuständigen Bewährungshelfer aufgebaut werden, um einen optimalen Betreuungsübergang der Inhaftierten gewährleisten zu können. In Bayern stellt das Übergangsmanagement somit einen wichtigen Bestandteil der vollzuglichen Behandlungsmaßnahmen dar. Wie Arloth treffend formuliert, hängt der Erfolg dieses Übergangsmanagements im Wesentlichen von der personellen und sachlichen Ausstattung in der Vollzugspraxis ab. ${ }^{13}$

Gemäß den Qualitätsstandards der Sozialdienste der Justizvollzugsanstalten in Bayern ist Übergangsmanagement die „konkrete Entlassungsvorbereitung“, die spätestens drei Monate vor dem voraussichtlichen Entlassungstermin beginnt und an den individuellen Bedarf des Gefangenen anzupassen ist. Als allgemeines Qualitätsmerkmal des Übergangsmanagement wird die „bei Bedarf einzuleitende Vernetzung mit den jeweils relevanten Hilfesystemen außerhalb des Vollzugs (staatliche Stellen, freie Träger, Ehrenamtliche)" angeführt. ${ }^{14}$

\section{Forschungsstand zur Haftentlassung}

Trotz der bereits angesprochenen von Problemen und Unsicherheiten begleiteten Entlassungsphase (sog. Entlassungsloch) liegen zur situativen Haftentlassung aus dem Jugendstrafvollzug in Deutschland nur wenige Studien vor. ${ }^{15}$ In der Regel wird in diesem Zusammenhang eher der Frage der Legalbewährung nach Haftentlassung nachgegangen. ${ }^{16}$ Die bedeutendste Untersuchung hinsichtlich der Entlassungsphase ist die Hanover Prison Study ${ }^{17}$ des $\mathrm{KFN}^{18}$ sowie deren Teilstudien, die darauf hinweisen, dass hier ein erheblicher Verbesserungsbedarf besteht.

Für die Hanover Prison Study wurden über 2400 Erstverbüßer im Alter von 14 bis 24 Jahren zu drei Zeitpunkten im Haftverlauf interviewt. Die Ergebnisse dieser Untersuchung zeigen insbesondere, dass die Wohnungs- und Arbeitssituation bei Haftentlassung sowie die Möglichkeiten des Bezugs von Sozialleistungen ungeklärt blieben. ${ }^{19}$ 59\% der jungen Menschen verließen die Haftanstalt ohne einen gesicherten Arbeits- oder Ausbildungsplatz oder auch nur die Aussicht darauf. 40\% der Befragten hatten an keiner Entlassungsvorbereitungsmaßnahme teilgenommen und von denjenigen, die einer sol-

12 http://www.dvjj.de/themenschwerpunkte/jugendstrafvollzug/mindeststandards-f-r-den-jug endstrafvollzug, zuletzt geprüft am 06.12.2013.

13 Arloth 2010, 347.

14 Bayerisches Staatsministerium der Justiz und für Verbraucherschutz 2011, 62.

15 Pruin 2011, 692.

16 Hosser u.a. 1997, 11.

17 http://www.kfn.de/Forschungsbereiche_und_Projekte/Abgeschlossene_Projekte/Entwicklu ngsfolgen_der_Jugendstrafe.htm, zuletzt geprüft am 06.12.2013.

18 Kriminologisches Forschungsinstitut Niedersachsen.

19 Hosser u.a. $2007403 \mathrm{ff}$. 
chen Maßnahme beiwohnten, bewerteten über 30\% diese als wenig hilfreich. Dementsprechend groß waren die Ängste der Inhaftierten, nach der Entlassung keinen Arbeitsoder Ausbildungsplatz zu finden und die damit verbundene Befürchtung, ihren Lebensunterhalt nicht bestreiten zu können. ${ }^{20}$

$\mathrm{Zu}$ ähnlichen Ergebnissen gelangt auch die längsschnittliche Interviewstudie von $\mathrm{Be}$ reswill \& Koesling, eine Teilstudie der Hanover Prison Study, für die 43 Häftlinge des Jugendstrafvollzugs zu verschiedenen Haftzeitpunkten befragt wurden. Die Ergebnisse belegen, dass sich viele der Entlassenen im Hinblick auf die vor ihnen liegenden Aufgaben überfordert fühlten. Das Gefühl der Überforderung könne vor allem auf eine unzureichende Unterstützung und Kooperationslücken zwischen Strafvollzug und den betreuenden Stellen außerhalb der Haftanstalten zurückgeführt werden ${ }^{21}$ und habe nicht selten das Misslingen des individuell zu bewältigenden Übergangs zur Folge. ${ }^{22}$

Die vorliegenden Studien weisen also insgesamt auf eine ausbaufähige Entlassungsvorbereitung hin. Die Evaluationen einzelner Modellprojekte ${ }^{23}$ zeigen, dass ein strukturierter, begleiteter Übergang aus der Haft in die Freiheit positive Effekte für die Haftentlassenen mit sich bringt. Ein Beispiel hierfür ist etwa das baden-württembergische Pilotprojekt BASIS. Junge Straftäter erhalten im Rahmen dieses Projekts unter anderem strukturierte Lernprogramme, Entlassungsbegleitung und Nachsorge. Die wissenschaftliche Begleitforschung zeigte keine Neuverurteilungen nach Haftentlassung innerhalb der Experimentalgruppe und es gelang eine bessere Integration in den Leistungsbereich. ${ }^{24}$ Zudem ergaben sich wesentliche Unterschiede zwischen Teilnehmern am Projekt BASIS und anderen Häftlingen unter anderem in den Bereichen „Beschäftigung nach der Haft“, „Erstellung von Bewerbungsunterlagen“ und „Verfügen über eine Ausbildungsstelle zum Entlasszeitpunkt“. ${ }^{25}$ Auch Untersuchungen aus dem Ausland, wie etwa die Evaluation der „Boston Reentry Initiative“ 26 oder des Wiedereingliederungsprogramms „Pathfinder", 27 belegen die positiven Effekte eines betreuten Haftübergangs auf die Rückfälligkeit von Haftentlassenen. ${ }^{28}$

Im Folgenden wird anhand qualitativer Daten die Umsetzung der Entlassung aus dem Jugendstrafvollzug in Bayern näher betrachtet. Der Fokus liegt auf dem geforderten Betreuungsübergang von der jeweiligen JVA zur Bewährungshilfe und der subjektiven Bewertung der Entlassungssituation einzelner Haftentlassener.

20 Dies. 2007, 409.

21 Bereswill u.a. 2007, $300 \mathrm{ff}$.

22 Bereswill 2010, $551 \mathrm{f}$.

23 Begleitforschung zu Maßnahmen im Bereich des Übergangsmanagements fehlt bisher weitestgehend (Pruin 2013, 692).

24 Löprick 2007, $441 \mathrm{ff}$.

25 Pruin 2013, $700 \mathrm{f}$.

26 Braga u.a. Journal of Research in Crime and Delinquency 46 (2009), 411, $420 \mathrm{ff}$.

27 Lewis u.a. Criminology \& Criminal Justice 7 (2007), 33, $46 \mathrm{f}$.

28 Goodstein/Sontheimer Criminal Justice and Behavior 24 (1997), 332, 351 ff.; Fagan Justice Quarterly 7 (1990) 233, 250 ff.

NK 26. Jg. 3/2014 


\section{E. Untersuchung}

Im Rahmen einer empirischen Erhebung zur Wahrnehmung der Umsetzung der gesetzlichen Vorgaben von Erziehungsmaßnahmen im Jugendstrafvollzug wurden fünf Bewährungshelfer und fünf aus dem Jugendstrafvollzug entlassene Probanden, die sich in ihrer Bewährungszeit befanden, unter anderem zu ihren Erfahrungen mit dem Betreuungsübergang bzw. der Entlassungssituation befragt. Darüber hinaus wurde Interviewmaterial aus einer weiteren Untersuchung ${ }^{29}$ verwendet, für die Interviews mit 18 jugendlichen und heranwachsenden Intensiv- und Mehrfachtätern mit zum Teil mehrfacher Hafterfahrung geführt wurden. Die Befragungen erfolgten mittels leitfadengestützter Interviews.

In den Interviews mit den Bewährungshelfern wurde insbesondere die vorgesehene Kooperation mit den Justizvollzugsanstalten im Vorfeld der Entlassung thematisiert. Die Haftentlassenen hingegen wurden gebeten, ihre Betreuung bei Haftentlassung darzulegen. Bei den ehemaligen Inhaftierten handelte es sich um nach dem Jugendstrafrecht verurteilte Jugendliche, Heranwachsende und junge Erwachsene mit erheblicher Vorbelastung insbesondere im Bereich der Gewalt- und/oder Sexualstraftaten.

Mittels der qualitativen Daten soll die subjektive Einschätzung des Übergangsmanagements und der Entlassungsvorbereitung aus Sicht einzelner Bewährungshelfer und aus dem Jugendstrafvollzug entlassener Probanden dargelegt werden. Da es sich um eine kleine Stichprobe handelt, sind keine verallgemeinerungsfähigen Aussagen zum Zustand der Haftentlassungsvorbereitungen in Bayern möglich, sondern lediglich vorsichtige Bewertungen der Umsetzung der gesetzlichen Vorgaben.

\section{F. Darstellung der Ergebnisse}

\section{Sicht der befragten Bewährungshelfer}

Die gemäß Art. 175 Abs. 4 BayStVollzG vorgesehene frühzeitige Zusammenarbeit zwischen Justizvollzug und Bewährungshilfe bei vorgesehener Haftentlassung ist aus Sicht der befragten Bewährungshelfer nur unzureichend umgesetzt. So erhält die Bewährungshilfe von der jeweiligen Justizvollzugsanstalt ein Schreiben mit der Bitte, die beruflichen und wirtschaftlichen Verhältnisse sowie die Beschaffenheit des sozialen Empfangsraums zu klären. In der Praxis werden diese Auskünfte telefonisch bei den Familien der Inhaftierten eingeholt, da die Kapazitäten der Bewährungshilfe nicht ausreichen, um sich ein eigenes Bild von der Situation zu verschaffen. Daher könnten diese Auskünfte ebenso gut mittels Selbstauskunftsbögen von den Familien bei Besuchen in der JVA eingeholt werden, so der Vorschlag von Seiten der Bewährungshilfe.

Weiterhin gaben die befragten Bewährungshelfer durchwegs an, dass die Zusammenarbeit mit den Justizvollzugsanstalten eher mäßig verlaufe und eine Intensivierung wünschenswert sei. Häufig werde die Bewährungshilfe erst unmittelbar vor oder bereits nach

29 http://www.mpicc.de/ww/de/pub/forschung/forschungsarbeit/kriminologie/rubikon.htm. 
Haftentlassung über das Haftende der Probanden informiert. Dies stößt insbesondere bei Endstrafenverbüßung auf Unverständnis von Seiten der interviewten Bewährungshelfer, da in diesen Fällen der Entlassungstermin hinreichend bekannt ist. Auch bei Probanden, die der Anweisung, bereits im Hafturlaub bei der Bewährungshilfe vorstellig zu werden, folgen, besteht in der Regel ein Zuständigkeitsproblem. Mangels Kenntnis von Entlassungsdatum und Fallübergabe zum Entlassungszeitpunkt ist kein spezifischer Bewährungshelfer für den jeweiligen Probanden zuständig. Einen direkten Betreuungsübergang von der Justizvollzugsanstalt zur Bewährungshilfe gebe es daher nur in Einzelfällen.

Die befragten Bewährungshelfer waren sich zudem einig, dass die Vorbereitung der jungen Inhaftierten auf die Haftentlassung mangelhaft sei. So gab ein Bewährungshelfer an, dass die Unterstützung der Delinquenten durch den Sozialdienst der Justizvollzugsanstalten im Hinblick auf die Wohnraumbeschaffung umfangreicher sein müsste, vor allem wenn eine Rückkehr in die Familie ausgeschlossen sei. In diesen Fällen wäre es oft nicht vermeidbar, die jungen Menschen in eine Pension oder Obdachloseneinrichtung zu übergeben. Darüber hinaus wurde bemängelt, dass die Organisation zahlreicher Angelegenheiten der Inhaftierten (etwa Beschaffung von Personal-, Sozialversicherungsausweis oder Lohnsteuerkarte), für die bereits im Haftverlauf Sorge getragen werden könnte, während des Strafvollzugs unterbleibe.

\section{Sicht der befragten Haftentlassenen}

Auch die 23 interviewten ehemaligen Inhaftierten bewerteten die Vorbereitung auf die Haftentlassung eher negativ. Die überwiegende Mehrheit der Befragten $(n=17)$ gab an, auf die Haftentlassung nicht vorbereitet worden zu sein. Ein Interviewter berichtete zudem, mehrfach um Hilfe für verschiedene Lebensbereiche gebeten, diese jedoch nicht erhalten zu haben. Dieser Proband empfand insbesondere die geplante Wohnsituation bei Entlassung so unangenehm, dass er es nach eigener Angabe vorgezogen hätte, inhaftiert zu bleiben. Weiterhin gab dieser Befragte an, seine Bedenken seien lediglich mit einem „das schaffen Sie schon“ abgetan worden. Die übrigen sieben Interviewten berichteten, sowohl während als auch nach der Haft von ihren Familien unterstützt worden und nach der Entlassung in ihr Elternhaus oder aber zu ihren Ehefrauen zurückgekehrt zu sein. Daher hätten sie von Seiten der Justiz keine Unterstützung beim Haftübergang benötigt. Jedoch handelt es sich bei der Rückkehr in die Familie um einen potenziell kriminogenen Faktor, der bereits vor der eigentlichen Inhaftierung wirksam gewesen sein könnte. Insbesondere bei jungen Mehrfachauffälligen bestehen häufig gravierende Problemlagen im familiären Bereich. ${ }^{30}$ Daher könnte der familiäre Empfangsraum ggf. auch einen negativen Einfluss ausüben. ${ }^{31}$

Zehn Befragten wurde vor Entlassung Hafturlaub gewährt, wobei der Ausgang in einem dieser Fälle so terminiert wurde, dass die Haftentlassung des Befragten einen Tag 
vorgezogen wurde. Eine solche Vollzugslockerung dürfte kaum dem „Entlassungsloch“ entgegenwirken und verfehlt die Zielsetzung von Vollzugslockerungen. Lockerungen sollen dazu dienen, sowohl die soziale Unterstützung außerhalb der Haftanstalt im Haftverlauf aufrecht zu erhalten als auch in der Haft erworbene Kenntnisse zu Verhaltensweisen im Leben außerhalb der Haft zu erproben. Damit leisten sie einen wesentlichen Beitrag zur Rückfallprävention. ${ }^{32}$ Kontakt zur Bewährungshilfe vor Haftentlassung entstand bei fünf Befragten.

15 Interviewte beschrieben die Zeit nach der Haftentlassung als schwer zu bewältigen. In diesem Zusammenhang gaben manche Befragte an, dass sie unmittelbar nach der Entlassung das Leben in Freiheit negativer empfanden als das Leben in Haft, da sie sich in Freiheit nur schwer zurechtfinden konnten. Hierbei wurde insbesondere auch das junge Alter zum Zeitpunkt der Inhaftierung als problematischer Faktor kommuniziert, wie das folgende Interviewzitat verdeutlicht:

"Ich bin mit 17 rein [...] und bin 21 wieder raus. Ich hab irgendwie nie diesen Übergang gehabt so ins Selbständige. [...] Woher sollte ich wissen, wie man sich 'nen Perso beantragt? Woher sollt ich wissen, wie man des macht, wie man jenes macht? [...]. Ich hab davor [vor der Inhaftierung (Anmerkung d. Verf.)] meine Zeit mit Drogen verbracht, dann bin ich irgendwann reingekommen, und dann komm ich raus und bin plötzlich erwachsen und muss mich wie ein erwachsener Mensch benehmen."

Es zeigt sich, dass die befragten jungen Menschen die Entwicklungsschritte, die sie in Freiheit hätten durchlaufen können, vermissen. Dadurch scheint sich eine Unsicherheit im Umgang mit Alltäglichem aufzubauen, die vermutlich daher rührt, dass für den Umgang mit diesen alltäglichen Dingen keine Strategien erlernt werden konnten. Ferner wurde wiederholt angegeben, dass die Erinnerung an das Hafterleben nach kurzer Zeit verblasst und damit auch sämtliche gute Vorsätze in Vergessenheit geraten, zumal der Gedanke an eine erneute Inhaftierung nicht mehr abschreckend gewesen sei.

\section{G. Diskussion}

$\mathrm{Zu}$ analysieren ist nun, welche Rückschlüsse anhand der Untersuchungsergebnisse auf die Umsetzung des Übergangsmanagements im Jugendstrafvollzug in Bayern gezogen werden können. Bei der Untersuchung handelt es sich, wie bereits erwähnt, nicht um eine Bestandsaufnahme der tatsächlichen Haftentlassungssituation, sondern um Einschätzungen einzelner Befragter anhand eigener Erfahrungen bei der Haftentlassung bzw. in der Berufspraxis.

Die dargestellten Ergebnisse weisen darauf hin, dass die gemäß Art. 79 BayStVollzG vorgesehene Hilfe, die den Gefangenen vor der Entlassung bei der Ordnung ihrer persönlichen, wirtschaftlichen und sozialen Angelegenheiten zuteilwerden sollte, bislang nicht in der vorgesehenen Weise umgesetzt wird und daher weiter ausgebaut werden sollte. Besonders da die Entlassungsvorbereitung der Gefangenen nach dem Verständnis

32 Subling ZJJ 19 (2008), 330, $333 \mathrm{f}$.

THEMEN 
des bayerischen Justizvollzugs am ersten Tag der Inhaftierung beginnt, ${ }^{33}$ wäre ein stärkerer Fokus auf die Organisation der Angelegenheiten der Inhaftierten im Haftverlauf möglich.

Auch die in Art. 175 Abs. 4 BayStVollzG und Nr. 7.1.1.2 BewHBek festgelegte frühzeitige Zusammenarbeit mit der Bewährungshilfe scheint den befragten Bewährungshelfern zufolge allenfalls in Einzelfällen stattzufinden. Dabei würde diese Kooperation in Fällen der sekundären Strafaussetzung die Grundlage für einen reibungslosen Betreuungsübergang vom Vollzug zur Bewährungshilfe im Sinne des Übergangsmanagements bilden. Hierdurch könnte ein wesentlicher Beitrag zur angestrebten Eindämmung des „Entlassungslochs“ geleistet und die Wiedereingliederungschancen verbessert werden. ${ }^{34}$ Jedoch scheinen nach wie vor zahlreiche Faktoren einer Verdichtung der Zusammenarbeit entgegenzuwirken. Zum einen weist der Informationsfluss vor Haftentlassung Lücken auf, zum anderen scheinen auch organisatorische Hindernisse, wie etwa die nicht geregelte Zuständigkeit eines konkreten Bewährungshelfers vor Haftentlassung und der damit einhergehende Mangel eines direkten Ansprechpartners, eine erfolgreiche Schnittstellenarbeit in diesem Bereich zu hemmen.

Die überwiegende Mehrheit der befragten Entlassenen fühlte sich nach der Haftentlassung auf sich allein gestellt, sofern keine familiäre Unterstützung bei der Regelung ihrer Angelegenheiten vorhanden war. Dabei weisen insbesondere junge Gewalt- und Sexualstraftäter einen besonders hohen Unterstützungsbedarf auf und sollten aufgrund ihres hohen Risikopotentials zwingend überwacht werden. ${ }^{35}$ Trotz der kleinen Stichprobe der Befragung könnte man darauf schließen, dass die gesetzlichen Vorgaben für Haftentlassungsvorbereitungen aus dem bayerischen Jugendstrafvollzug nicht im erforderlichen Maße umgesetzt werden; gerade da der besondere Bedarf der von der Untersuchung erfassten Gewalt- und Sexualstraftäter durchaus erkannt wird. ${ }^{36} \mathrm{Zu}$ diesem Befund gelangen auch Bereswill E Koesling sowie Hosser E Lauterbach für den Jugendstrafvollzug anderer Bundesländer. ${ }^{37}$

Dabei sind eine adäquate Entlassungsvorbereitung sowie eine intensive Nachsorge besonders im Jugendstrafvollzug erforderlich, um die Vollzugsziele erreichen zu können und die Lebensbedingungen der jungen Menschen an diesem kritischen Übergang zu

33 Arbeitsgruppe „Übergangsmanagement“ 2012, 9.

34 Dünkel 1999, 125.

35 Arbeitsgruppe „Übergangsmanagement“ 2012, 7 (Obwohl der Begriff „Risikopotential“ im Bericht der Arbeitsgruppe „Übergangsmanagement" wiederholt Verwendung findet, wird er darin nicht definiert. Es wird davon ausgegangen, dass unter Risikopotential nicht das Rückfallrisiko von Gewalt- und Sexualstraftätern zu verstehen ist, da die einschlägigen Rückfallraten nach den meisten Sexual- und Gewaltstraftaten eher gering sind (Jeble u.a. 2013, 113 ff.; Jeble u.a. 2010, 118 ff.). Anscheinend ist für das Risikopotential eines Straftäters die Qualität eines einschlägigen Rückfalldelikts ausschlaggebend. Das Risikopotential bezeichnet daher wohl die potentielle Gefährlichkeit, die bei einschlägiger Rückfälligkeit von Sexual- und Gewaltstraftätern für mögliche Opfer entsteht, die seelisch oder körperlich schwer geschädigt werden könnten.).

36 Dies., 7.

37 Bereswill u.a. 2007, 299 ff.; Hosser u.a. 2007, 403 ff.

NK 26. Jg. $3 / 2014$ 
verbessern. ${ }^{38}$ Sowohl anhand der Aussagen der befragten Bewährungshelfer als auch aus der Sicht der befragten Haftentlassenen können vorsichtige Rückschlüsse auf die Umsetzung der gesetzlichen Vorgaben und den Stand des angestrebten Übergangsmanagements gezogen werden bzw. auf die subjektive Realität der Befragten, deren Problematik wichtige Ansatzpunkte für die Betreuung in der Übergangsphase bei Haftentlassung liefern. Die Untersuchung liefert Hinweise darauf, dass aus Sicht der jungen Haftentlassenen das Übergangsmanagement noch nicht in ausreichendem Maße durchgeführt zu werden, sondern vielmehr auf wenige Einzelfälle beschränkt zu sein scheint. Wenn davon ausgegangen wird, dass der Erfolg des Übergangsmanagements im Wesentlichen von der personellen und sachlichen Ausstattung in der Vollzugspraxis abhängt, ${ }^{39}$ so scheint eine Anpassung der Ausstattung notwendig, um das Übergangsmanagement nicht nur in Einzelfällen zu einen Bestandteil der vollzuglichen Behandlungsmaßnahmen werden zu lassen.

\section{Literatur:}

Arbeitsgruppe „Übergangsmanagement" (2012) Optimierung des Übergangsmanagements in den bayerischen Justizvollzugsanstalten, Bericht der Arbeitsgruppe „Übergangsmanagement“. Abrufbar unter: http://www.justiz.bayern.de/media/pdf/ueberga ngsmanagement.pdf

Arloth (2008) Strafvollzugsgesetz, Kommentar, 2. Aufl.

Arloth (2010) Zur weiteren Entwicklung des Strafvollzugs, in: Dölling / Albrecht (Hrsg.), Verbrechen - Strafe - Resozialisierung, Festschrift für Schöch, 337

Babru.a. Successful Reentry: What Differentiates Successful and Unsuccessful Parolees?, in: International Journal of Offender Therapy and Comparative Criminology 54 (2010), 667-692

Barton Incorporating the Strengths Perspective into Intensive Juvenile Aftercare, in: Western Criminology Review 7 (2006), 48-61

Bayerisches Staatsministerium der Justiz und für Verbraucherschutz (2011) QualitätsHandbuch, Standards und Qualitätssicherung für die Sozialdienste bei den Justizvollzugsanstalten in Bayern

Bereswill u.a. (2007) Brüchige Erfolge - Biographische Diskontinuität, Inhaftierung und Integration, in: Goerdeler / Walkenhorst (Hrsg.), Jugendstrafvollzug in Deutschland, Neue Gesetze, neue Strukturen, neue Praxis?, 294

38 Babr u.a. International Journal of Offender Therapy and Comparative Criminology 54 (2010), 667, 689 f.; Dünkel/Geng 2006, 2; Barton Western Criminology Review 7 (2006), 48, 51; Wößner u.a. 2013, $664 \mathrm{ff}$.

39 Arloth 2010, 347. 
Bereswill (2007) "Von der Welt abgeschlossen", Die einschneidende Erfahrung einer Inhaftierung im Jugendstrafvollzug, in: Goerdeler / Walkenhorst (Hrsg.), Jugendstrafvollzug in Deutschland, Neue Gesetze, neue Strukturen, neue Praxis?, 163

Bereswill (2010) Strafhaft als biographischer Einschnitt. Befunde zum Jugendstrafvollzug aus der Perspektive der Insassen, in: Dollinger, Handbuch Jugendkriminalität, 545

Braga u.a. Controlling Violent Offenders Released to the Community: An Evaluation of the Boston Reentry Initiative, in: Journal of Research in Crime and Delinquency 46 (2009), 411-436

Cottonaro / Subling. (2007) Weichenstellung im Betreuungskontinuum: Diagnose, Prognose, Indikation und Vollzugsplanung, in: Goerdeler / Walkenhorst (Hrsg.), Jugendstrafvollzug in Deutschland, Neue Gesetze, neue Strukturen, neue Praxis?, 222

Dünkel (1999) Jugendstrafvollzug zwischen Erziehung und Strafe, Entwicklungen und Perspektiven im internationalen Vergleich, in: Feuerhelm / Böhm (Hrsg.), Festschrift für Böhm, 99

Dünkel / Geng (2006) Aktuelle rechtstatsächliche Befunde zum Jugendstrafvollzug in Deutschland. Ergebnisse einer Erhebung bei den Jugendstrafanstalten zum 31.1.2006. Abrufbar unter: http://www.rsf.uni-greifswald.de/fileadmin/mediapool/lehrstuehle/d uenkel/Duenkel_Geng_ZJJ.pdf

Fagan Treatment and reintegration of violent juvenile offenders: Experimental results, in: Justice Quarterly, 7 (1990), 233-263

Goodstein/Sontheimer The implementation of an intensive aftercare program for serious juvenile offenders, in: Criminal Justice and Behavior, 24 (1997), 332-359

Hosser u.a. (1997) Gefängnis und die Folgen, Identitätsentwicklung und kriminelles Handeln Jugendlicher und Heranwachsender während und nach einer Jugendstrafe

Hosser u.a. (2007) Und was kommt danach? Entlassungsvorbereitung und Nachentlassungssituation junger Strafentlassener, in: Goerdeler / Walkenhorst (Hrsg.), Jugendstrafvollzug in Deutschland, Neue Gesetze, neue Strukturen, neue Praxis?, 396

Jeble u.a. (2010) Legalbewährung nach strafrechtlichen Sanktionen. Eine bundesweite Rückfalluntersuchung 2004 bis 2007. Abrufbar unter: http://www.bmj.de/SharedDocs/ Downloads/DE/pdfs/Legalbewaehrung_nach_strafrechtlichen_sanktionen_2004_2007 .pdf?_blob=publicationFile

Jeble u.a. (2013) Legalbewährung nach strafrechtlichen Sanktionen. Eine bundesweite Rückfalluntersuchung 2007 bis 2010 und 2004 bis 2010. Abrufbar unter: http://www.b mj.de/SharedDocs/Downloads/DE/pdfs/Legalbwaehrung_nach_strafrechtlichen_Sank tionen_2007_2010_u_2004_2010.pdf;jsessionid=6F8E2FBA8E9AED7290A0349EA1B 0976C.1_cid334?_blob=publicationFile

Lewis u.a. What works in resettlement? Findings from seven Pathfinders for short-term prisoners in England and Wales, in: Criminology \& Criminal Justice 7 (2007), 33-53 
Löprick (2007) Übergang aus der Haft in die Freiheit - Ein Beispiel aus dem Offenen Jugendvollzug Göttingen, in: Goerdeler / Walkenhorst (Hrsg.), Jugendstrafvollzug in Deutschland, Neue Gesetze, neue Strukturen, neue Praxis?, 436

Matt Übergangsmanagement, Zur Konzeption einer systematischen Wiedereingliederungsstrategie von (Ex)Strafgefangenen und Straffälligen, in: NK 22 (2010), 34-39

Matt Übergangsmanagement, in: Zeitschrift für Jugendkriminalrecht und Jugendhilfe, 22 (2011), 422-428

Obder (2009) "Intensivtäter" - ein neuer Tätertypus?, in: Bindel-Kögel (Hrsg.), Jugendliche Mehrfach- und "Intensivtäter", Entwicklungen, Strategien, Konzepte, 17

Pruin (2011) Die Evaluation des Projekts BASIS in der JVA Adelsheim, in: Stelly / Thomas (Hrsg.), Erziehung und Strafe, Symposium zum 35-jährigen Bestehen der JVA Adelsheim, 159

Pruin (2013) Übergangsmanagement im Jugendstrafvollzug: Die Evaluation des Projekts BASIS in der JVA Adelsheim, in: Dölling / Jehle (Hrsg.), Täter Taten Opfer, Grundlagenfragen und aktuelle Probleme der Kriminalität und ihrer Kontrolle, 691

Schwedler (2013) Zum Umgang mit Sexualstraftätern nach ihrer Entlassung, in: Wößner u.a. (Hrsg.), Sexuelle Gewalt und Sozialtherapie: bisherige Daten und Analysen zur Längsschnittstudie „Sexualstraftäter in den sozialtherapeutischen Abteilungen des Freistaates Sachsen“, 189

Stelly u.a. (2003) Verlaufsmuster und Wendepunkte in der Lebensgeschichte, Eine Untersuchung des Einflusses soziobiographischer Merkmale auf sozial abweichende und sozial integrierte Karrieren, Bd. 2

Subling Sozialtherapie im Jugendstrafvollzug: Prinzipien wirksamer Behandlung, in: Zeitschrift für Jugendkriminalrecht und Jugendhilfe, 19 (2008), 330-335

Wößner u.a. (2013) Sozialtherapie im Jugendstrafvollzug - und dann?, in: Dölling / Jehle (Hrsg.), Täter Taten Opfer, Grundlagenfragen und aktuelle Probleme der Kriminalität und ihrer Kontrolle, 643

Kontakt:

Maria Walsh, M.A.

wissenschaftliche Mitarbeiterin

Abteilung Kriminologie des Max-Planck-Instituts

für ausländisches und internationales Strafrecht

Günterstalstraße 73

79100 Freiburg

m.walsh@mpicc.de 